\title{
The Roll of the Entrepreneur in the Establishment of Economic Equilibria
}

\author{
Er'el Granot
}

Additional information is available at the end of the chapter

http://dx.doi.org/10.5772/intechopen.70674

\begin{abstract}
In this chapter, a simple market model is presented to illustrate how random entrepreneurial activity can be responsible for the establishment of economic equilibria without the assumption of perfect knowledge. In this model it is assumed that the entrepreneurs (both traders and producers) have no information regarding the other entrepreneurs' preferences, wealth, or production skills. The only information they have is the past transaction prices, and yet this information is sufficient for the market to reach equilibrium price. Equilibrium is not a stationary process on the microscopic level. It is a process, which consists of interactions between entrepreneurs, who act randomly without insight. Consequently, the market price continuously oscillates randomly around the equilibrium values. The higher the risk the producers are willing to take, the more stable is the equilibrium. When entrepreneurial actions are depressed, the market may drift from its optimal point. This model also investigates the more realistic scenario, in which, due to specialization, the production boundary frontiers are convex (instead of linear). It is shown that in this case, the drifts are suppressed and the optimal equilibrium is more stable. Moreover, the amount of risk aversion has a clear effect on the production growth of the economy. The lower the risk aversion is, the higher is the growth rate of the economy.
\end{abstract}

Keywords: entrepreneurship, market equilibrium, entrepreneurial behavior, economic equilibria, entrepreneur, iterative economic processes, Austrian school of economics

\section{Introduction}

The entrepreneur is usually regarded as a unique special individual, who has some foresight abilities. Usually, entrepreneurship is related to the introduction of a new technology, a new method, and a new invention, and therefore its presence imbalances the established economy, forcing it to migrate from one equilibrium to the next. Since the traditional Marshallian-Walrasian economic analysis is based on equilibrium, no room was left to the entrepreneur in this traditional economic paradigm (see [1, 2]; see also [3] where the term entrepreneur is used for small 
business manager rather than an actor in an uncertain economic world). Since entrepreneurs interrupt the equilibrium state, they were no more than noises (or as Kirzner calls: pests) to the well-established economic theories.

The neoclassical economic equilibrium is established by perfect competition, as was formulated by Knight (see Ref. [4]), and perfect knowledge. It is therefore recognized that the participating actors act as if they possess all the information regarding the market $[3,4]$. Clearly, they do not really have perfect knowledge, however, to all practical matters; it was conjectured that they act as if they do.

The Austrian school of economics based its theory on ordinary men and ordinary man's behavior and therefore rejected these prepositions [5, 6], and yet they recognized that the market converges to an equilibrium [7]. This observation was regarded by Hayek as one of the most fundamental laws of economics [6, 7].

The mechanism, by which the market is in a permanent convergence process toward equilibrium, despite the lack of knowledge, is based on entrepreneurial action [8, 9]. The information is transmitted via the entrepreneurial action, where every act toward equilibrium is encouraged, while every act away from equilibrium is discouraged via the profit and loss mechanism. Therefore, entrepreneurs are crucial ingredients in the process toward equilibrium, but as far as I know, this process has never been demonstrated.

Despite the fact that the Austrian economists recognized that the information is transmitted via the price mechanism, they still attributed insight to the entrepreneurial act $[8,9]$. Such an attitude makes it difficult to establish a scientific analysis of the connection between the entrepreneurial behavior and the convergence to equilibrium. However, this conjecture is superfluous. Since the profit and loss mechanism is an essential ingredient in the process, it can be the only necessary feedback mechanism, and no additional conjectures (as entrepreneurial insights or the assumptions that errors lure the entrepreneurs) are needed.

A physical analogy to such a process is diffusion. In the microscopic realm, diffusion occurs by random movements and collisions of microscopic particles. However, in the macroscopic level, the diffusion equation emerges, which frustrates any deviation from the equilibrium values. On the macroscopic level, there are no signs of the complex randomness of the microscopic world.

This physical analogy can be useful to illustrate the problems in the Austrian picture of the equilibrium state. According to their view, at the state of equilibrium, all expectations are coordinated in a perfect concert. As Kirzner put it, "(in the state of equilibrium) All plans are made in the correct expectation of the corresponding plans being made by others. No one's plan is frustrated on account of others failing to act as that plan had anticipated they would act. No realised plan is regretted as having failed to make use of opportunities, made possible by the actions of others, which hindsight reveals but which foresight failed to anticipate" [8].

Going back to the physical analogy, at a state of equilibrium, a gas occupies a container homogenously. Using Kirzner's (or Hayek's) terminology, one can state that every particle is completely coordinated with the rest of the particles, since every particle is, on the average, propagated toward the vacancies that the other particles have generated. After all, on average 
the gas is at rest. However, it is clear that no information is transmitted between the particles. They all propagate in a totally random manner. In this case, all the "information" that a single particle needs to know about the gas to reach equilibrium is the average volume of the gas, which in this case is merely the dimension of the vessel. The particle does not need to know any information about the other particles, not even their total number.

In a similar manner economic equilibrium can be generated in a gradual process of random events, where every one of the actors has only a rough estimate of some average property of the market.

Ever since the establishment of the Marshallian synthesis [2], one of the fundamental tools in the neoclassical theory is the equilibrium between demand and supply.

This tool was frequently used by the Austrian school of economics as well; however, they regarded it with much suspicion since they did not recognize supply as a separate entity, which depends on objective costs (as the classical economists conjectured), but as a realization of the demand to other commodities. That is, there is a supply of good A only since the supplier prefers other goods over good A. Therefore, in principle, the knowledge of the individuals' preference ranking or their utility functions (which can be shown to be equivalent [10]) is sufficient to determine the supply and demand curves, and therefore it can determine the equilibrium point.

Moreover, as was shown in Ref. [11], the willingness to trade depends not only on the price of the good but on the amount of exchanged goods as well. Therefore, the history of trading has an impact on the amount of the traded goods. If a person does not possess any units of good A, he may be willing to purchase 5 units, but if, let us assume, he already has 3 units, then he will not be willing to purchase the extra 2 units. This is indeed a plausible scenario, which emphasizes the fact that both curves have a "thickness" or a certain amount of uncertainty.

Another problem arises from the discretization of the commodities. For example, let the intersection between the demand and supply curves occurs at the price of 14.5 units of A, for which 23.5 units of B are traded. If these are indivisible goods (like cars or houses), then clearly no equilibrium can be established. For 23 units of B demand exceeds supply, and for 24 units supply exceeds demand. In such a scenario, it is clear that the system would oscillate around the unreachable equilibrium point. The mechanism, by which the system oscillates around this point, is the random entrepreneurial activity.

It is the object of this chapter to demonstrate that not only does the economic equilibrium can be established by entrepreneurial actions but also that these actions can be totally random. Nevertheless, we begin with stationary market analysis.

\section{Deriving the supply-demand intersection from the preference ranking matrices}

Let the market consists of $N$ traders. The $n$th trader possesses $a_{n}$ units of $\mathrm{A}$ and $b_{n}$ units of B. The $n$th trader's utility preference matrix is $R_{n}(a, b)[11]$. 
Therefore, every trader seeks to improve its state by increasing its preference ranking. That is, if initially his possessions of $\mathrm{A}$ and $\mathrm{B}$ are $a_{n}$ and $b_{n}$, respectively, then he will be willing to trade $\Delta a$ units of $\mathrm{A}$ for $\Delta b$ units of $\mathrm{B}$ (note that $\Delta a$ and $\Delta b$ can be negative) provided

$$
R_{n}\left(a_{n}-\Delta a, b_{n}+\Delta b\right)>R_{n}\left(a_{n}, b_{n}\right) .
$$

Thus, for every $n$, there are infinite preferred states $m$, which are characterized by $a_{m}$ and $b_{m}$ which obey

$$
R_{n}\left(a_{m}, b_{m}\right)>R_{n}\left(a_{n}, b_{n}\right)
$$

To upgrade to the preferred state $m, \Delta a=a_{n}-a_{m}$ units of A should be exchanged with $\Delta b=b_{m}-b_{n}$ units of $\mathrm{B}$ (or vice versa). Hence, a price can be determined

$$
p_{m} \equiv \frac{a_{n}-a_{m}}{b_{m}-b_{n}} .
$$

Then, for any price $p$, the maximum units of B that the $n$th trader is willing to purchase is

$$
\Delta b_{n}(p)=\max _{p_{m}>p}\left\{b_{m}-b_{n}\right\}
$$

(where the subscript stands for the fact that the maximum operation is acting on the group of states $m$, for which $R_{n}\left(a_{m}, b_{m}\right)>R_{n}\left(a_{n}, b_{n}\right)$ and $\left.p_{m} \equiv \frac{a_{n}-a_{m}}{b_{m}-b_{n}}>p\right)$.

Therefore, the total demand of units of $\mathrm{B}$, i.e., $b_{\text {Demand }}(p)$ for the price $p$, is

$$
b_{\text {Demand }}(p)=\sum_{n} \Delta b_{n}(p)=\sum_{n} \max _{p_{m}>p}\left\{b_{m}-b_{n}\right\}
$$

Similarly, on the supply side, the maximum units of B that the $n$th trader is willing to sell is

$$
\Delta b_{n}(p)=\max _{p_{m}<p}\left\{b_{n}-b_{m}\right\},
$$

and then the total supply of units of B, i.e., $b_{\text {Supply }}(p)$ for the price $p$, is

$$
b_{\text {Supply }}(p)=\sum_{n} \Delta b_{n}(p)=\sum_{n} \max _{p_{m}<p}\left\{b_{n}-b_{m}\right\} .
$$

Since the equilibrium price $p^{*}$ corresponds to the intersection point of the two curves, then

$$
b_{\text {Supply }}\left(p^{*}\right)=b_{\text {Demand }}\left(p^{*}\right) \text {. }
$$

\section{Perpetual dynamic convergence to equilibrium}

Clearly, such a mechanism can work only in trading arenas, in which the preferences of each trader are publicly published. An approximation of such a trading arena is the modern stock 
exchanges. But these are only approximations; in ordinary markets, the trader's preference is not a public knowledge, and it takes an entrepreneurial activity to unveil it.

First, the trader needs to contact a different trader, and usually this part of the process is a pseudorandom one.

Let trader $q$ has $a_{q}$ units of $\mathrm{A}$ and $b_{q}$ units of $\mathrm{B}$, and similarly trader $p$ has $a_{p}$ and $b_{p}$ units of $\mathrm{A}$ and $\mathrm{B}$, respectively. Then, their initial preference ranking is $R_{q}\left(a_{q}, b_{q}\right)$ and $R_{p}\left(a_{p}, b_{p}\right)$, respectively. From this initial condition, the bargaining stage begins.

In this stage they seek $\Delta a$ and $\Delta b$ such that

$$
\begin{gathered}
R_{q}\left(a_{q}+\Delta a, b_{q}-\Delta b\right)>R_{q}\left(a_{q}, b_{q}\right) \text { and } \\
R_{p}\left(a_{p}-\Delta a, b_{p}+\Delta b\right)>R_{p}\left(a_{p}, b_{p}\right) .
\end{gathered}
$$

If they do not find such numbers, i.e., they do not agree on the price and the amount of exchanged goods [11], then they look for a different trading partner. In this case, there is no entrepreneurial activity since they have nothing to lose by stopping the bargaining (since the other trader offers only to reduce their preference status).

However, if there exist $\Delta a$ and $\Delta b$ that meets these demands, then the entrepreneurial stage begins since each one of them has to decide whether he should or should not take the deal. In this case he chooses an entrepreneurial act because he takes risks. He is risking in losing the good deal in front of him.

The method, by which the trader (now entrepreneur) can evaluate how beneficial is the deal, is by comparison of previous transactions. The entrepreneur must have a memory or some kind of a record of historical prices.

The entrepreneurial process is therefore a continuous process, in which the entrepreneur seeks to improve his future condition by acting today based on his past experience.

In many respects this process resembles the process, in which, according to Mises [12], money regains its value. Mises solved Helfferich vicious cycle (see, e.g., [13]), by explaining that money has a current value, i.e., people want it today, since they expect that other people would still want money tomorrow on the account of their yesterday's experience.

In all these cases, the individual takes a risk, which makes him an entrepreneur. His decision is based on the two questions: what kind of information he possesses and how large a risk is he willing to take?

The decision can be based on published public information of previous transactions. In many markets the past transactions are published. If not all the information is distributed among the participants, then at least part of it is. However, even if the past transactions and prices are classified, or at least, are inaccessible, then the trader can base his decision on his own trading experience. In this case, the convergence is slower but provided the traders choose their trading partner randomly, the process can still converge. 
As for the risk taken, the entrepreneur has to decide on the margins of the acceptable price. That is, if $\left\langle p_{\text {previous }}\right\rangle$ is the mean value of the previous transaction, then the entrepreneur has to decide on $\Delta p$, which determines his tolerance to price fluctuations.

If the trader is a seller then $\Delta p$ determines a minimum acceptable value for future transaction prices

$$
p_{\text {new }}>\left\langle p_{\text {previous }}\right\rangle-\Delta p
$$

Similarly, if the trader is a buyer, then $\Delta p$ determines a maximum acceptable value for future transaction prices

$$
p_{\text {new }}<\left\langle p_{\text {previous }}\right\rangle+\Delta p
$$

The lower $\Delta p$ is, the higher is the risk the entrepreneur is willing to take. When $\Delta p$ has high positive values, the trader is a risk averse actor, while $\Delta p$ has low (or negative) values, the trader is a risk-seeker.

In particular if the transaction price is distributed normally with a mean $\left\langle p_{\text {previous }}\right\rangle$ and standard deviation $\sigma$, then the trader takes a risk, which can be quantified by the probability

$$
\frac{1}{2} \operatorname{erfc}\left(\frac{\Delta p}{\sigma}\right)
$$

(where $\operatorname{erfc}(x)$ is the complementary error function [14]) to reject the transaction.

It should be stressed, however, that in reality not only does not the trader have the distribution, but he cannot even tell whether there is one. In the simulation (in the following section), no such knowledge was assumed.

For simplicity, it is assumed that all the traders have the same utility function $R$, in which case

$$
R(a, b)=-(a-105)^{2}-(b-105)^{2}, a \leq 100 \text { and } b \leq 100 .
$$

The market in this simulation consists of $N=1000$ traders, where half of them initially have $a_{n}=40 \pm 2$ units of $\mathrm{A}$ and none of $\mathrm{B}$ and half of them possess $b_{n}=70 \pm 2$ units of $\mathrm{B}$ and none of A.

The demand and supply curves of this market are presented in Figure $\mathbf{1}$ (on the left), and as can be seen, the equilibrium price is around $p^{*} \sim 0.75$.

In Figure 1 (on the right), the result of a dynamic market simulation is plotted. In every iteration, two traders are chosen: the first from the owners of As and the second from the owners of Bs. First, they both check if trading is beneficial for both of them, i.e., that their status after trading is better off than their current status (according to the utility function). Then, they both check if the price agrees with their risk aversion. In this simulation, it was taken that $\Delta p$ is about half the standard deviation of previous price transactions, i.e., 

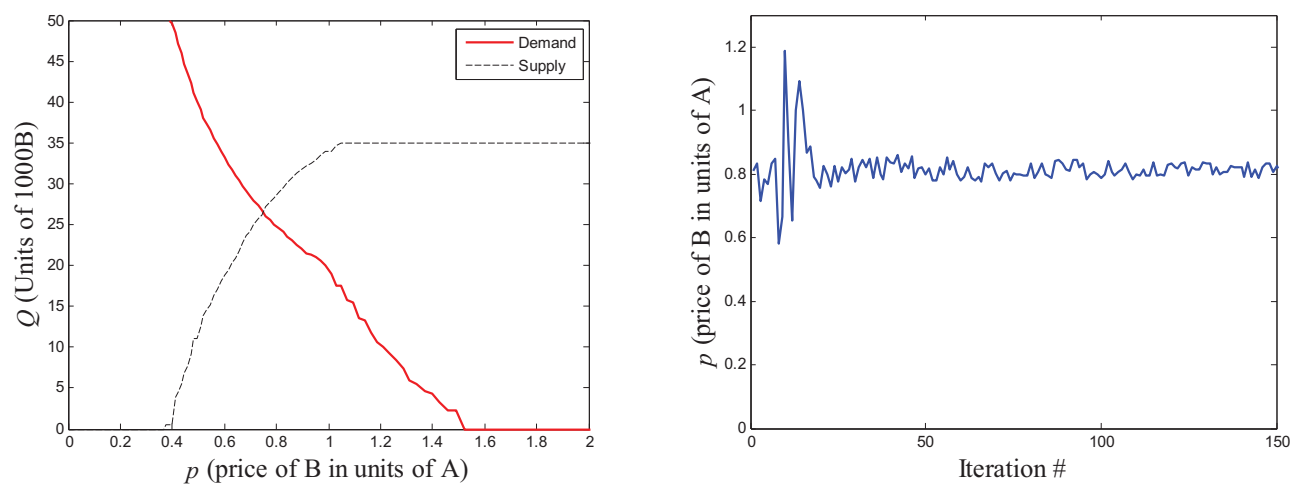

Figure 1. Simulation results. Left: static analysis of demand-supply curve intersection. Right: a dynamic market-the price variations vs. the iteration number.

$$
\Delta p=c \sqrt{\left\langle p_{\text {previous }}^{2}\right\rangle-\left\langle p_{\text {previous }}\right\rangle^{2}} \text {, where } c=0.5
$$

The transaction will take place only if: (1) The transaction improve their preference ranking, and that (2) The transaction price agrees with their risk aversion.

As can be shown from this simulation, the prices oscillate but quickly converge to the average value $p^{*} \sim 0.8$, which is close but not identical to the demand-supply intersection point. The small disagreement can be attributed to the fact that the supply and demand curves were calculated under the assumption that the traders would prefer to trade the largest amount of goods, which, as was explained in [11], this is not always the case. Moreover, dynamic convergence has a memory, and it depends on the initial state, which is randomly selected, in this simulation.

Moreover, we see that the system does not converge to a single value but keep oscillating randomly around the mean value. When $\Delta p$ decreases, the convergence is quicker but the amount of exchanged goods (and the number of transaction) decreases as well.

\section{Entrepreneurship in production}

In trading, entrepreneurship is relatively simple. There are only two relevant parameters: the price and the amount of exchange goods. However, if the traders are producers as well, they have to decide about additional parameters: the amount of goods to produce. This is a much more risky operation. In trading, the entrepreneur can decide whether to make the trade or to reject it. In the latter, he may put himself at a risk of losing a good deal for the prospect of finding a better one. However, by rejecting a deal, his state remains intact. Neither has it improved nor worsened.

When the entrepreneur decides to change his production strategy, he put himself in a larger risk of finding himself in a worse position. The reason is that the production abilities are 
bounded by his production frontiers, and therefore should he decides to increase the production of good A, then a corresponding reduction in the production of good B is obligatory. By doing so he temporarily reduces his optimal production point.

By increasing the production of goods, he does not need, and simultaneously decreasing the production of goods he does need, the producer specializes.

However, essentially the two entrepreneurial actions (trading and production) are similar. In both cases his decisions depend on the other producers' preferences. The problem is, of course, that he does not know the preference ranking of the other buyers (producers).

In the simulation that follows, we will demonstrate that the price system solves this problem.

In the simulation, each one of the producers has a production frontier, which he cannot exceed. Let $b_{n}[a]$ be the production frontier of the $n$th producer, i.e., while producing $a$ units of $\mathrm{A}$, he cannot produce more than $b_{n}[a]$ units of $\mathrm{B}$.

In the case of linear production frontiers, i.e., if the nth producer's production is limited by

$$
\frac{a}{A_{n}}+\frac{b}{B_{n}} \leq 1
$$

then

$$
b_{n}[a]=B_{n}\left(1-a / A_{n}\right)
$$

However, it can be generalized to any form of production frontier. In particular, it can be used even when specialization is taken under considerations [11].

Then, initially, before the traders begin their mutual interaction, every producer produces according to its preference ranking $R_{n}(a, b)$. Thus, initially

$$
R_{n}^{(0)}=R_{n}\left(a_{n}^{(0)}, b_{n}^{(0)}\right)=\max _{b<b_{n}\left[a_{n}\right]} R_{n}(a, b)
$$

i.e., $R_{n}^{(0)}$ is the highest ranking of producer $n$, which he reaches when he possess $a_{n}^{(0)}$ and $b_{n}^{(0)}$ units of $\mathrm{A}$ and $\mathrm{B}$, respectively.

When the producers begin to interact, i.e., begin to trade, then initially when there is no single price in the market, they just bargain, but they do not have a relative price for comparison. However, as was explained in the previous section, eventually a price is determined, and the market is cleared. Thus, an iterative process begins.

Let $m$ stands for the iteration number. Initially $m=0$.

Once the price is determined, then it can be used in the entrepreneurial process of the next iteration.

If the price is determined by equating supply and demand (like in the stock exchange) 


$$
b_{\text {Supply }}\left(p^{(m)}\right)=b_{\text {Demand }}\left(p^{(m)}\right)
$$

where the superscript $(m)$ stands for the $m$ th iteration, while the $n$th producer produced $a_{n}^{(m)}, b_{n}^{(m)}$ in the $m$ th iteration, then in the next iteration, he can randomly change these amounts a little to

$$
\begin{aligned}
& a_{n}^{(m+1)}=a_{n}^{(m)}+\delta a_{n}^{(m)} \\
& b_{n}^{(m+1)}=b_{n}^{(m)}+\delta b_{n}^{(m)},
\end{aligned}
$$

where $\delta a_{n}^{(m)}$ and $\delta b_{n}^{(m)}$ are random deviations under the constrains

$$
b_{n}\left[a_{n}^{(m+1)}\right]<b_{n}^{(m+1)}
$$

and

$$
a_{n}^{(m+1)} \geq 0, \quad b_{n}^{(m+1)} \geq 0
$$

Then, the producer checks, not that the state $\left\{a_{n}^{(m+1)}, b_{n}^{(m+1)}\right\}$ is preferred over the state $\left\{a_{n}^{(m)}, b_{n}^{(m)}\right\}$ because clearly it is not, but whether there is a third state that he can reach by trading, provided that the price is lower than $p^{(m)}$ (i.e., the price of the previous iterations). That is, he checks among all possible states $\{a, b\}$ that are reachable by trade, i.e., states which keep

$$
b-b_{n}^{(m+1)} \leq-p^{(m)}\left(a-a_{n}^{(m+1)}\right)
$$

the one with the highest ranking and compare it to his current ranking. If he finds improvement, i.e., if mathematically

$$
R_{n}\left(a_{n}^{(m)}, b_{n}^{(m)}\right)<\max _{b-b_{n}^{(m+1)} \leq-p^{(m)}\left(a-a_{n}^{(m+1)}\right)} R_{n}(a, b),
$$

then $\left\{a_{n}^{(m+1)}, b_{n}^{(m+1)}\right\}$ are chosen to be the next iteration production goals; otherwise, this trial is rejected, and the previous iteration production goals $\left\{a_{n}^{(m)}, b_{n}^{(m)}\right\}$ are kept.

Clearly, the fact that the producer evaluated that there is a state $\{a, b\}$, which can be reached by trading, doesn't mean that it will be reached. There are other players in this system, who also change gradually the amount of their production strategies. But the previous price is the only information that the traders can use to improve their production strategies. As can be seen from Eqs. (22)-(25), the traders use the price of the $m$ th iteration to improve their production judgment regarding the next, $(m+1)$ th one. 


\section{Simulation of entrepreneurial activity in production}

We used a simulation to illustrate the market process with the presence of entrepreneurial activity.

In the simulation the market consists of $N=800$ producers with the linear production frontier, i.e., Eqs. (16) and (17) are used when $A_{n}$ and $B_{n}$ have normal distribution with mean 85 and standard deviation 15 with a maximum value of 100, and to emphasize the effect of specialization, the ranking matrix (discrete utility function) was replaced with

$$
R_{n}(a, b)=-(a-105)^{4}-(b-105)^{4} \text { for } a \leq 100 \text { and } b \leq 100 .
$$

In every iteration the deviations $a_{n}^{(m)}, b_{n}^{(m)}$ were selected randomly with the probability

$$
p\left(a_{n}^{(m)}=q\right)=p\left(b_{n}^{(m)}=q\right)=\operatorname{erfc}\left(\frac{q+1 / 2}{Q}\right)-\operatorname{erfc}\left(\frac{q-1 / 2}{Q}\right) .
$$

where $q$ is an integer number. In this simulation $Q=80$.

The result of this simulation is presented in Figure 2. In this figure the initial (prior to trading) and final (after 200 iterations) distributions are presented in a false color presentation. As could be expected, since the ranking matrix is symmetric, most traders prefer not to give precedence of one commodity over the next. From the same reason, and due to the linear production frontier, there is no fundamental difference after trading takes place. Indeed, there are more producers, who prefer to deviate considerably from their initial state; however, as Figure 2 shows, they are the minority.

The dynamic of the process is illustrated in Figure 3. Despite the fact that there is no substantial difference in the market price, the gradual specialization increases the amount of exchange goods.

The effect of the specialization and the trading on the supply-demand curve is presented in Figure 4. While the intersection point remains at the price level $p \cong 1$, the amount of exchanged goods has increased considerably.
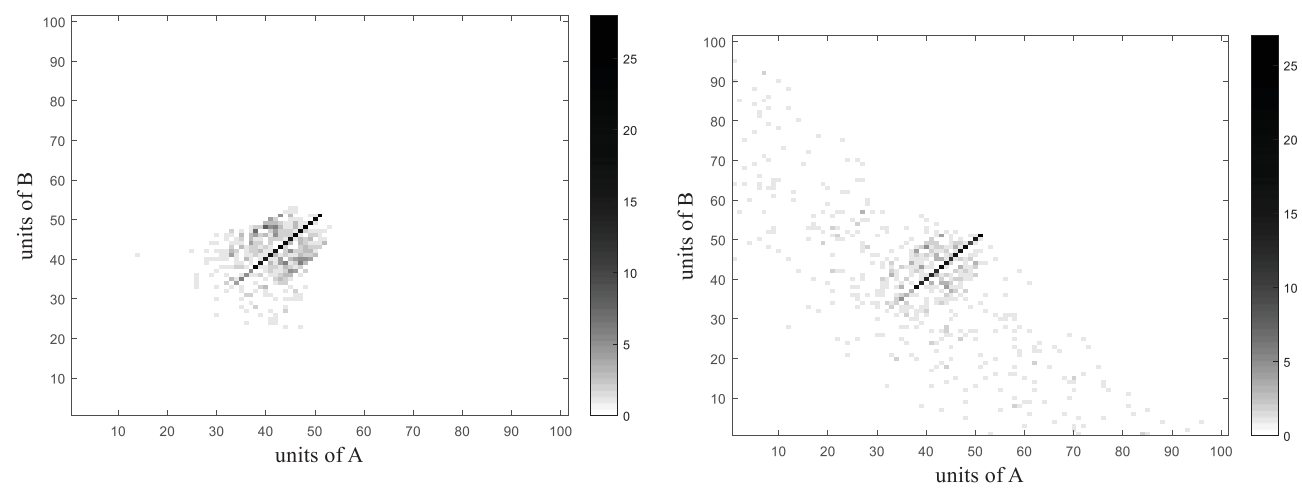

Figure 2. Left: a 2D histogram map of the producer's production after the first iteration. The false colors represent the number of producers. Right: after 200 iterations. 

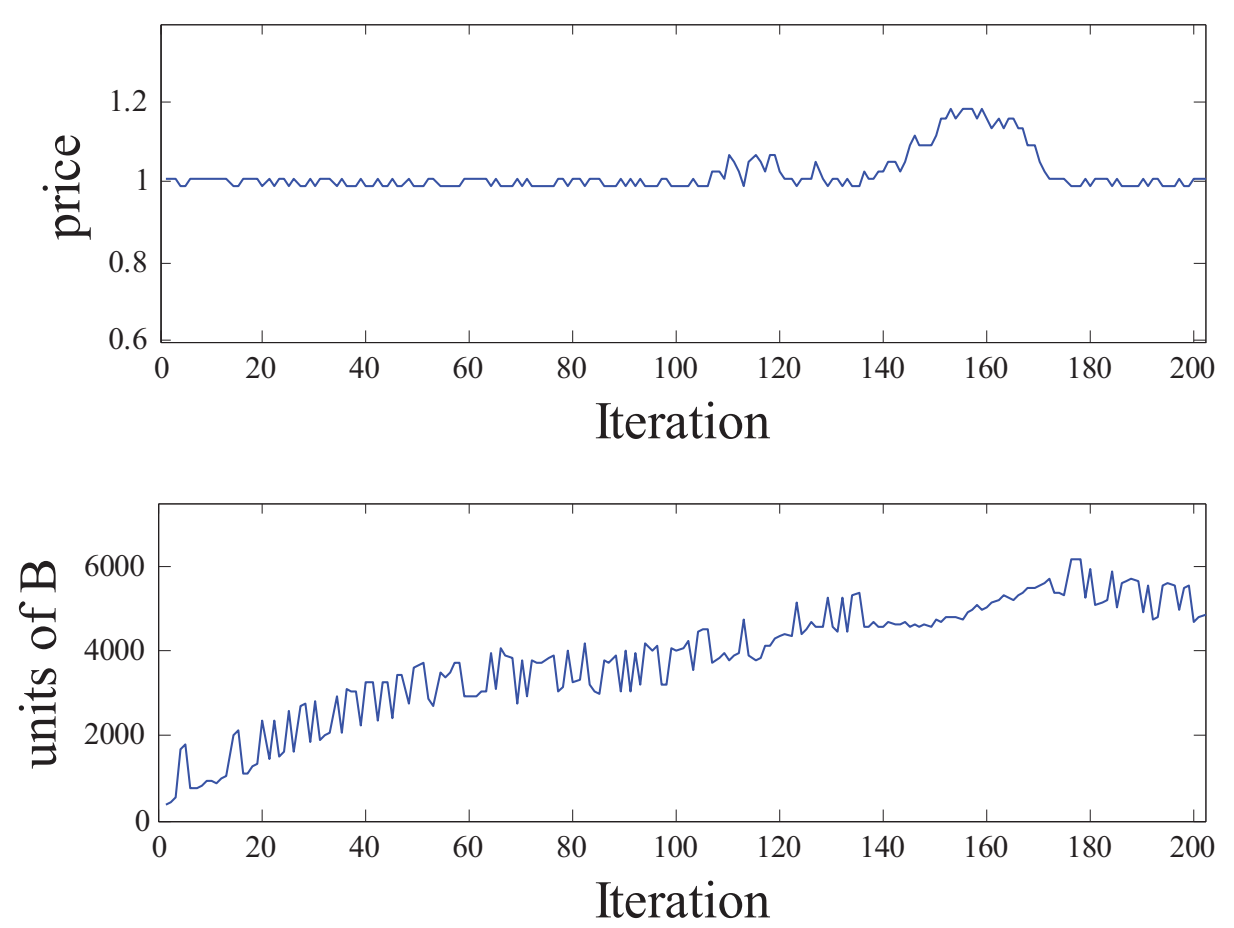

Figure 3. The temporal dependence of the market price of B in units of A (upper plot) and the amount of exchanged good (lower plot).

However, the dynamic results are different if the variations are considerably smaller. In Figures 5-7, the same simulation was taken for $Q=8.5$, in which case, the dynamic is much more affected by the initial state and the arbitrariness of the first random transactions.
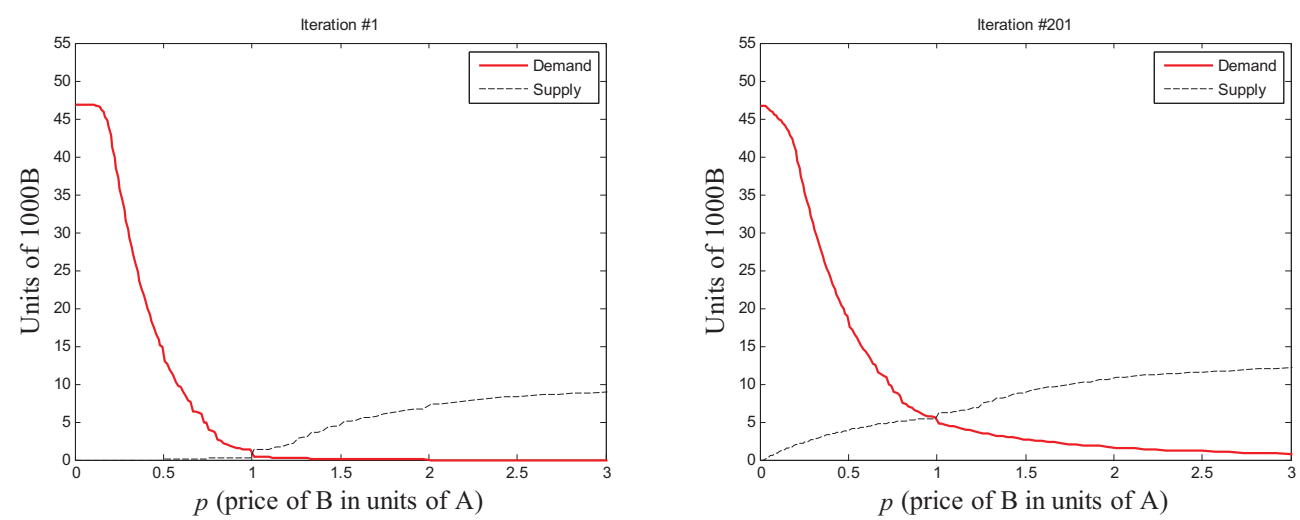

Figure 4. The supply-demand curves before trading and specialization (left) and after 200 trading iterations (right). 

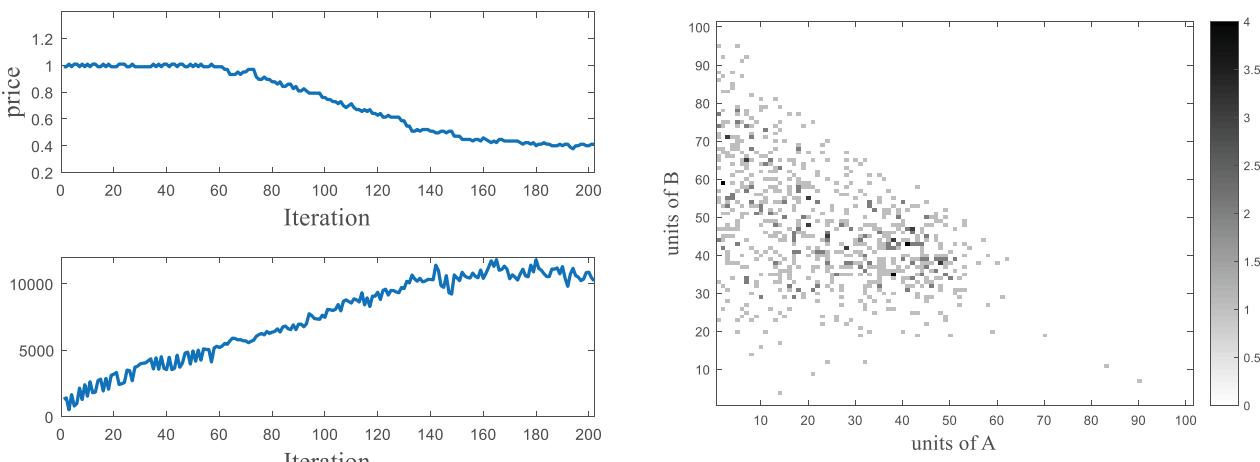

Figure 5. The temporal dependence of the market price and exchanged goods (left) and the distribution of the producers in the population after 200 iterations (right).
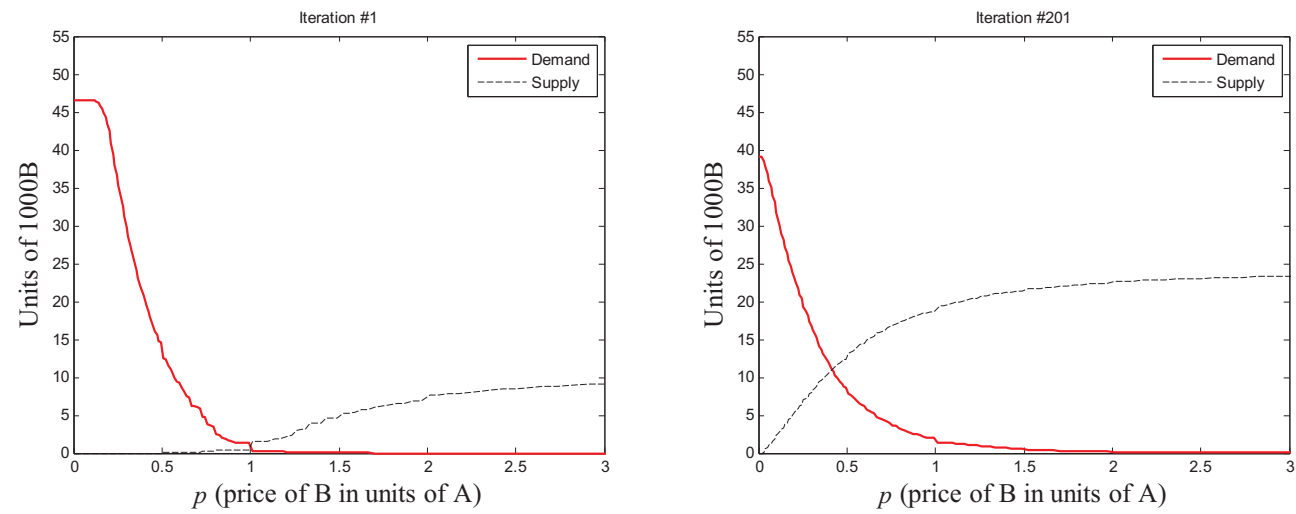

Figure 6. The supply-demand curves of the simulation of Figure 5 before trading (left) and after 200 iterations (right).
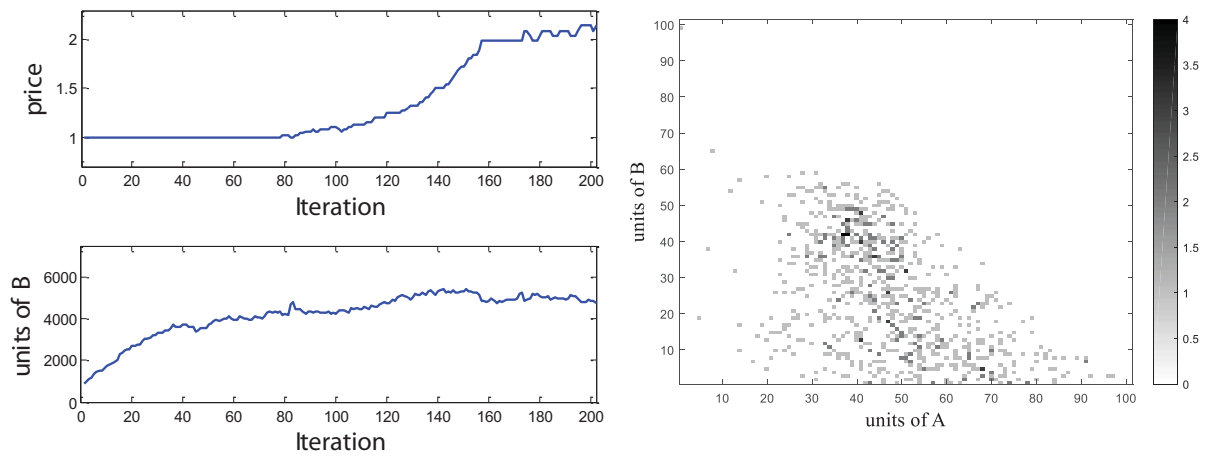

Figure 7. A different run of the same simulation that is generated in Figure 5. 
Consequently, the price may gradually drift to different values. To return to the preferred price $p=1$, the system has to experience a large perturbation, which has a very low probability in this case. Thus, as can be seen in Figure 5 after about 90 iterations, a perturbation causes a sudden drift toward lower prices. This behavior is also shown in the supply-demand curves in Figure 6 where in this case there is a clear change in the intersection point's price. As can be seen in Figure 7, a different run of the same simulation can present a drift toward higher prices. This is due to the sensitivity of the problem on the initial conditions (the population distribution) and on the specific trading decisions, which are also random.

\section{Specialization}

In the previous section, it was assumed that the production frontier is a linear function. This production frontier goes back to Ricardo's law of comparative advantage [15-17].

Despite its popularity [18-20], this production frontier ignores the effect of specialization. Superficially, it can be argued that if the producer is focused only on a single product, i.e., it produces only A or only B, then this producer is specializing in producing this single good. However, this analysis assumed that while he "specializes" in producing a single good, his production rate (production per units of time) was not improved. This assumption ignores the main feature of specialization, namely, that specialization increases the production rate (the production efficiency).

In Ref. [11] the effect of specialization on the law of association was presented. It this reference a mathematical presentation of the effect of specialization on the production frontier was introduced.

In general, the linear frontier was replaced with a convex one:

$$
\left(a / A_{n}\right)^{\alpha}+\left(b / B_{n}\right)^{\beta} \leq 1
$$

where $\alpha \leq 1$ and $\beta \leq 1$ are parameters that characterize the specialization in producing A and $\mathrm{B}$, respectively.

In case where $\alpha=\beta$, then this production frontier corresponds to the statement that specialization increases the good's production by a factor of (see Ref. [11])

$$
F=2^{1 / \alpha-1}
$$

\section{Simulation with specialization}

We now apply the simulation of the previous section on the following production frontier, which considers specialization:

$$
\left(a / A_{n}\right)^{0.5}+\left(b / B_{n}\right)^{0.5} \leq 1
$$

i.e., 


$$
b_{n}[a]=B_{n}\left(1-\sqrt{a / A_{n}}\right)^{2}
$$

with the same $A_{n}, B_{n}$, and $R_{n}(a, b)$ of the previous section. In this case specialization increases production, according to Eq. (29), by a factor of $F=2$.

As was demonstrated in Ref. [11], curved production frontier, i.e., Eq. (28), causes instability in the initial state even when all the producers have exactly the same production frontier (this is not the case when they all have the same linear, i.e., Eq. (16), production frontier). Therefore, specialization and trading are encouraged. Moreover, the curved frontier depresses drifts, since any small drift highly encourages entrepreneur to change their production strategies accordingly, and due to the curvature, even small drift directly affects the producers. As was shown in the previous section-this is not the case in the linear production frontier scenario. In Figures 8-10 the results of the simulation with $Q=80$ are presented. Similarly, the results of a similar simulation but with $Q=8.5$ are presented in Figures $\mathbf{1 1}$ and 12. As can be seen, unlike the linear production frontier
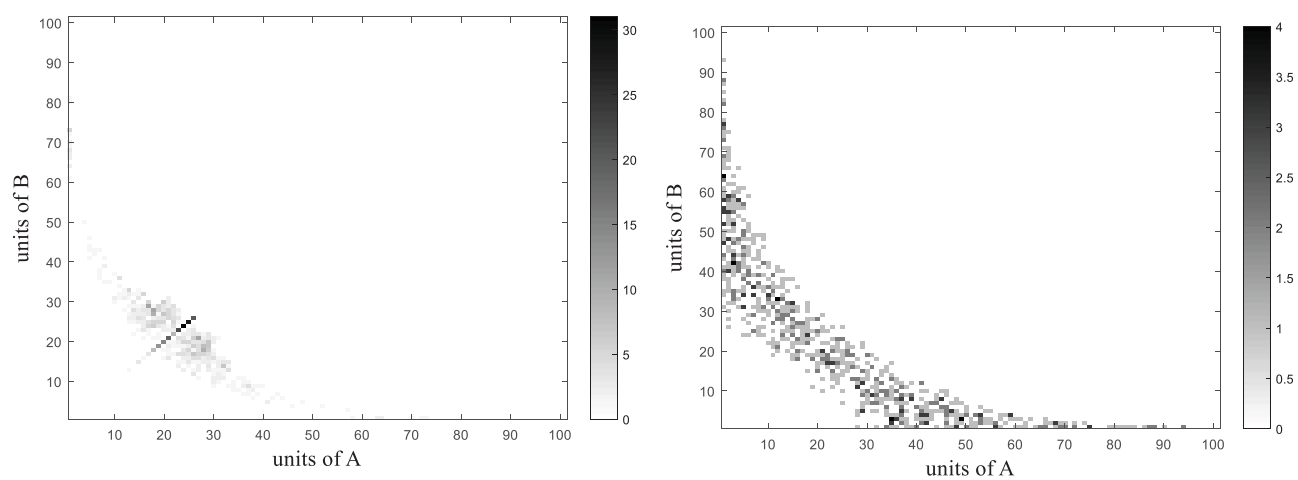

Figure 8. The effect of specialization on the producer's production histogram for $Q=80$. Left: after the first iteration. Right: 1400 iterations later. The false colors represent the number of producers.
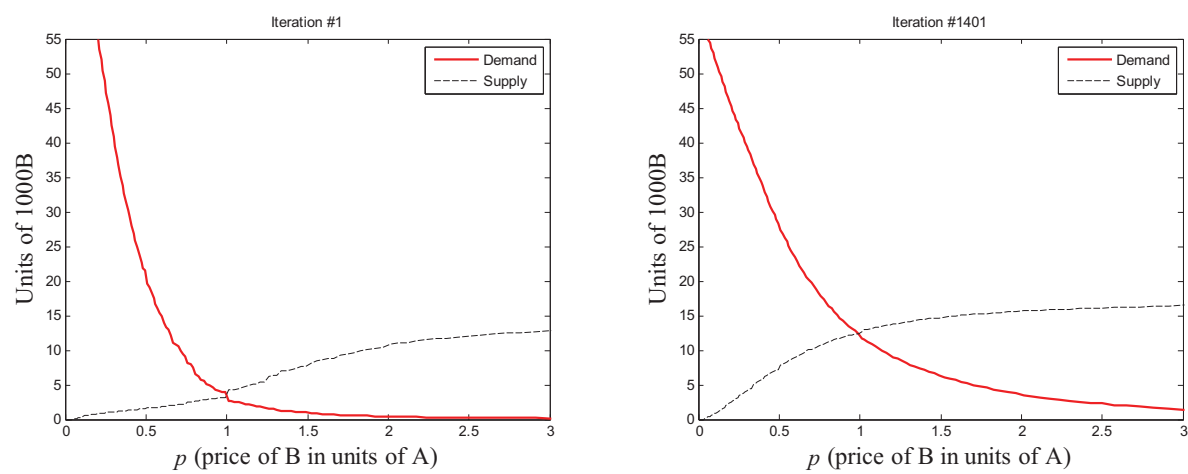

Figure 9. The supply-demand curves before trading and specialization (left) and after 1400 iterations (right). 

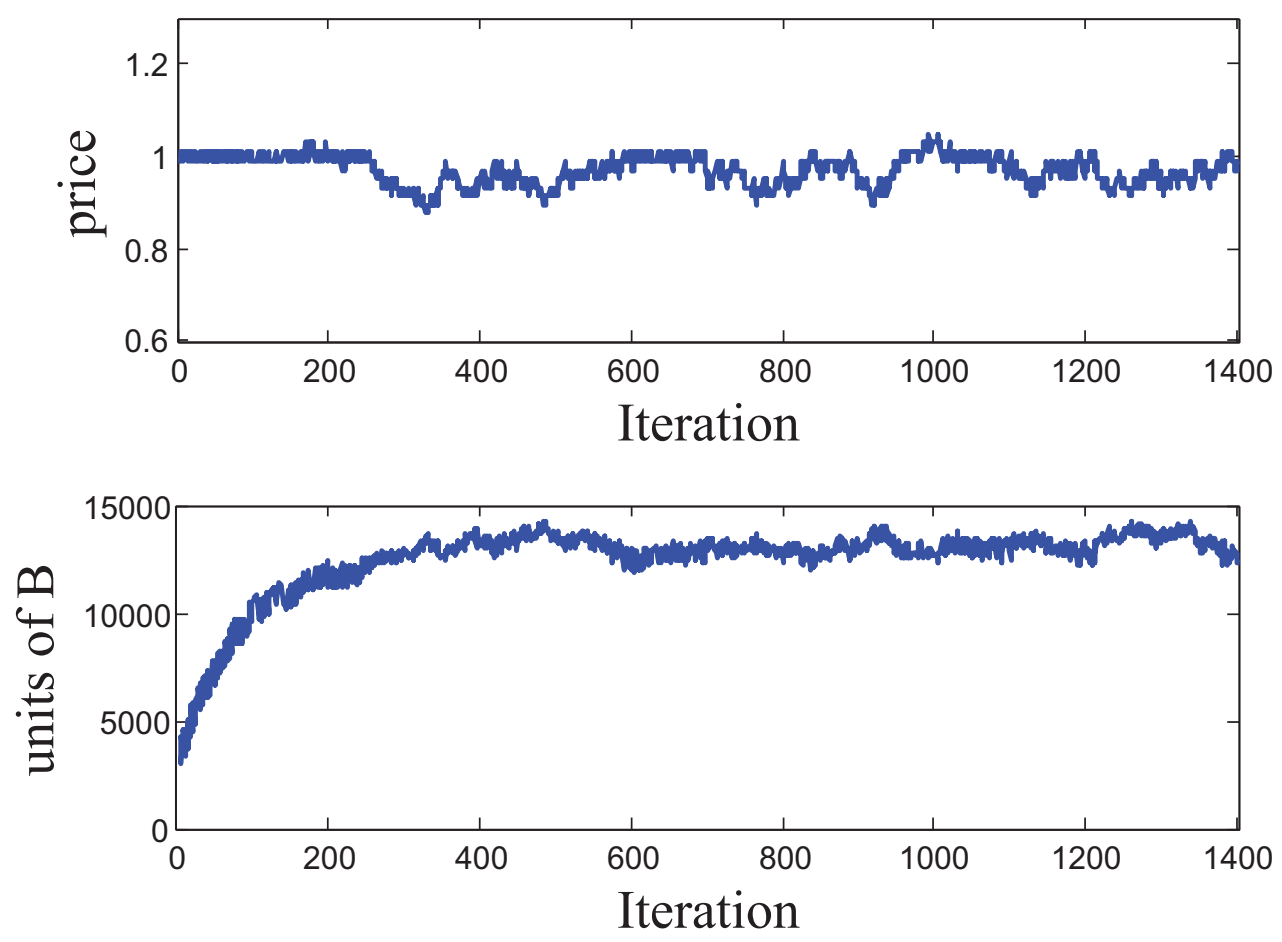

Figure 10. The temporal dependence of the market price (upper plot) and the amount of exchanged good (lower plot).
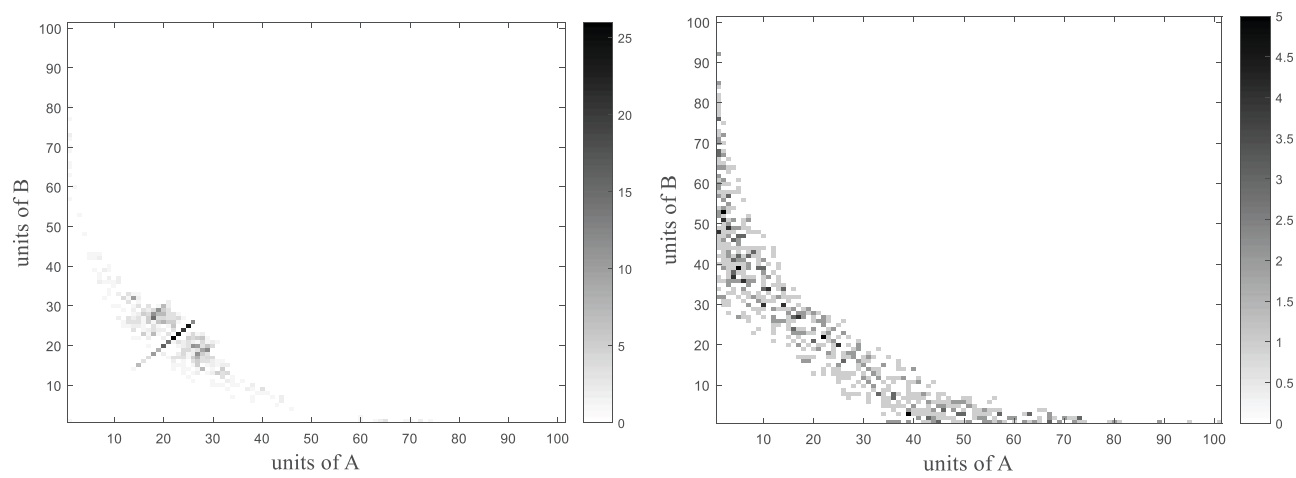

Figure 11. Same as in Figure 8 but with $Q=8.5$.

case, there is no qualitative difference between the two. The main difference is that large fluctuations (risk loving $Q=80$ ) help to keep the system at the optimal equilibrium value $(p=1)$, while smaller fluctuations (risk aversion $Q=8.5$ ) do it less efficiently, and small drifts last longer (more than 700 iterations when $Q=80$ instead of less than 100 when $Q=8.5$ ). 

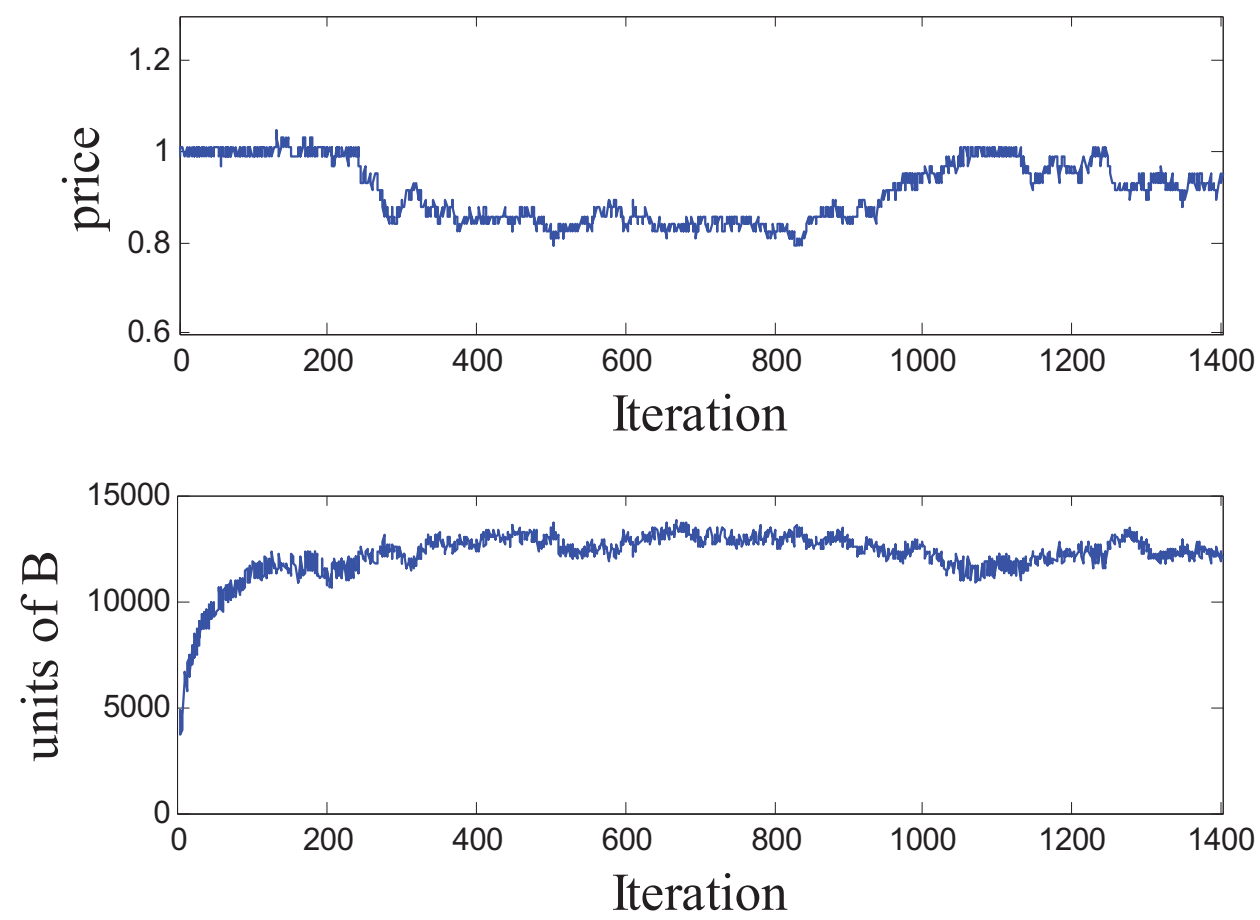

Figure 12. Same as in Figure 10 but with $Q=8.5$.

\section{Production growth and entrepreneurship}

In the previous section, we have seen that there is a major effect of the amount of risk the entrepreneurs are willing to take (which was quantified by the parameter $Q$ ) on the stability of the equilibrium. However, it seems that in this simplified model, the amount of risk taken is irrelevant to the final equilibrium case. Nevertheless, the rate toward equilibrium is clearly related to the entrepreneur risk aversion parameter $Q$. On the left side of Figure 13, the total production parameter ( $M$ is the total number of producer, and $p$ is the current market price)

$$
\text { production }=\sum_{n=1}^{M}\left(a_{n}+b_{n} p\right)
$$

is plotted for various values of $Q$ as a function of the iteration number for the first 24 iterations. As this plot clearly suggests, the higher the parameter $Q$, the faster is the production growth.

On the right side of Figure 13, the rate of the relative production growth (PG) $\frac{\Delta p}{p \Delta n}$ is measured as a function of the relative risk taken, i.e., as a function of the ratio between the production 

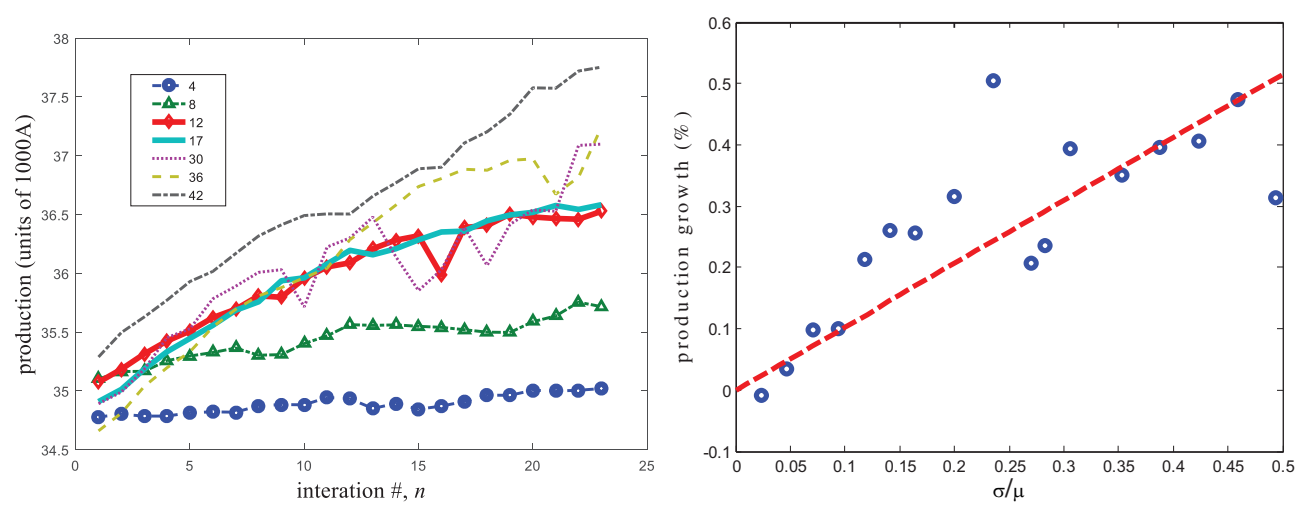

Figure 13. Left: the production as a function of the iteration number for different values of $Q$ (from 4 to 42 ). Right: the production growth rate as a function of $\sigma / \mu$ (which is a relative evaluation of the risk aversion).

standard deviation $\sigma(Q$, in the above examples), and the mean initial production $\mu$. This plot suggests the following simple linear relation:

$$
P G \cong C \frac{\sigma}{\mu}
$$

where $C=(1.0 \pm 0.2) \%$.

\section{Main conclusions}

A simple market model was presented to illustrate how the market reaches equilibrium and clearance without the assumption of perfect knowledge. In fact, the model assumed that the traders and producers have no information about other producers' preferences, wealth, or production skills, and yet their knowledge about the past transaction prices and their entrepreneurial activity keeps the market oscillates randomly near equilibrium.

The producers use the past transaction prices to make entrepreneurial decisions regarding their future production strategies.

The amount of risk aversion, which is quantified in a single parameter, determines how stable the equilibrium is. The lower the population's risk aversion, the more stable is the equilibrium.

When the production frontier is linear, and the producers are risk averse, the equilibrium price can drift from its optimal value. Future research is needed to investigate the dynamic of these drifts and their sensitivity on initial conditions.

Moreover, to emphasize the effect of specialization, a convex production frontier was simulated as well. In this case, the equilibrium prices are much more stable and are less affected by the producers' risk aversion. 
Nevertheless, the amount of risk aversion has a clear effect on the production growth of the economy. The lower the risk aversion is, the higher is the growth rate of the economy.

Another important general conclusion is that economic equilibrium is not a stationary process, at least not on the microscopic domain. It may be regarded as stationary only on the macroscopic level. Like molecule gas in a container, economic equilibria are generated by random interactions between entrepreneurs, which act without insight. Only on the macroscopic level, it seems as if deviations from equilibrium lure the actors (or particles) to correct the error.

\section{Author details}

Er'el Granot

Address all correspondence to: erel@ariel.ac.il

Department of Electrical and Electronics Engineering, Ariel University, Ariel, Israel

\section{References}

[1] van Daal J, Jolink A. Equilibrium Economics of Léon Walras. London: Routledge; 1993

[2] Marshall A. Principles of Economics, Great Minds Series. Prometheus Books; 1997

[3] Gravelle H, Rees R. Microeconomics. Harlow: Pearson Education; 2004

[4] Knight FH. Risk, Uncertainty and Profit. New York: Augustus M. Kelley; 1964

[5] von Mises L. Human Action: A Treatise on Economics. Ludwig von Mises Institute: Yale University Press; 1949

[6] Rothbard MN. Man Economy and State. 2nd ed. Scholar's Edition: Ludwig von Mises Institute; 2009

[7] Hayek FA. Individualism and Economic Order. Chicago: University of Chicago Press; 1958

[8] Kirzner IM. How market work: Disequilibrium, Entrepreneurship and Discovery. London: The Institute of Economic Affairs; 1997. p. 78

[9] Kirzner IM. Competition and Entrepreneurship. Chicago and London: The University of Chicago Press; 1973

[10] Debreu G. Representation of a Preference Ordering by a Numerical Function. In: Thrall, Davis, Coombs, editors. Decision Process. New York: Wiley; 1954. p. 159-165

[11] Granot E. Ricardo's law of comparative advantage and the Law of Association: A subjective analysis. Chapter 3 in Economics and Development. Rijeka: INTECH; 2017 
[12] von Mises L. The Theory of Money and Credit. Yale University Press; 1953

[13] McCulloch JH. Misesian Insights for Modern Macroeconomics. The Quarterly Journal of Austrian Economics. 2014;17:3-18

[14] Abramowitz M, Stegun IA. Handbook of Mathematical Functions. New York: Dover Publicationa; 1972

[15] Ricardo D. On the Principles of Political Economy and Taxation. UK: John Murray; 1817

[16] Maneschi A. Comparative Advantage in International Trade: A Historical Perspective. Cheltenham, UK: Edward Elgar; 1998

[17] Hollander S. The Economics of David Ricardo. Tronto Press: Toronto. Univ; 1979

[18] Rothbard MN. Classical Economics: An Austrian Perspective on the History of Economic Thought. Vol II: Edward Elgar Publishing, Cheltenham; 2006

[19] Krugman PR, Obstfeld M. Boston, MA. In: International Economics: Theory \& Policy, Addison-Wesley. Boston, 8th ed. 2009

[20] Murphy RP. Choice Cooperation. Independent Institute, Oakland: Enterprise and Human Action; 2015 
